\title{
MicroRNA-144 functions as a tumor suppressor in gastric cancer by targeting cyclooxygenase-2
}

\author{
QIANG YAO ${ }^{1}$, ANXIN GU $^{2}$, ZHUOZHONG WANG $^{3}$ and YINGWEI XUE ${ }^{1}$ \\ Departments of ${ }^{1}$ Gastrointestinal Surgery and ${ }^{2}$ Radiotherapy, Harbin Medical University Cancer Hospital; \\ ${ }^{3}$ Department of Statistics, Harbin Medical University, Harbin, Heilongjiang 150000, P.R. China
}

Received September 27, 2016; Accepted November 29, 2017

DOI: $10.3892 /$ etm.2018.5763

\begin{abstract}
Gastric cancer (GC) poses a serious public health threat and the 5-year survival rate of patients with GC is low. MicroRNAs (miRNAs/miRs) may serve oncogenic or tumor suppressor functions during tumorigenesis by regulating cell proliferation, apoptosis, migration and invasion and it has been demonstrated that they may be dysregulated in various types of cancer. The present study demonstrated that miR-144 and GATA4 were downregulated in GC tissues and cell lines and suggested that this may be due to hypermethylation. Additionally, miR-144 and GATA4 had synergistic effects on GC cells by repressing cell proliferation and inducing cell cycle arrest and apoptosis. The results of bioinformatics and a luciferase reporter assay indicated that cyclooxygenase-2 (COX-2) is a direct target of miR-144 and that miR-144 negatively regulated the expression of $\mathrm{COX}-2$, which inhibits the viability of GC cells. GATA4 also induced a similar effect on COX-2. Taken together, the results of the present study may improve understanding of the underlying mechanism of miR-144 and GATA4 in GC.
\end{abstract}

\section{Introduction}

Gastric cancer (GC), one of the leading causes of cancer-associated mortality, is a malignant epithelial cancer that poses a serious threat to public health, particularly in China $(1,2)$. Current treatments for GC depend on the tumor-node-metastasis stage, the type of cancer tissue (intestinal or diffuse) and the patient's physical condition $(3,4)$. It has been demonstrated that patients with early-stage GC have a longer survival time, due to them receiving prompt improved gastrectomy and radical therapy $(4,5)$. However, the overall prognosis for the disease remains poor, the 5 -year survival rate is $\sim 30 \%$ for patients not

Correspondence to: Mr. Yingwei Xue, Department of Gastrointestinal Surgery, Harbin Medical University Cancer Hospital, 150 Haping Road, Harbin, Heilongjiang 150000, P.R. China

E-mail: yingweix92@163.com

Key words: microRNA-144, GATA4, cyclooxygenase-2, gastric cancer with early-stage GC at the time of their initial diagnosis (6-8). Therefore, developing a predictive biomarker for the diagnosis and prognosis of GC may improve the targeted therapeutic options available to treat GC.

The dysregulation of microRNAs (miRNAs/miRs) may serve oncogenic or tumor suppressor functions in tumorigenesis, as miRNAs regulate cell proliferation, apoptosis, migration and invasion (9). Previous studies have suggested that miRNAs may be used as molecular biomarkers in the diagnosis and prognosis of GC (10-12). Oncogenic miRNAs, which are generally upregulated in GC, exert their function by silencing the expression of genes that inhibit the cell cycle and/or genes associated with apoptotic pathways (13-15). By contrast, miRNAs that are downregulated act as tumor suppressors during GC progression. These miRNAs generally exert their function via anti-proliferative and pro-apoptotic associated genes (14-16). Additionally, decreased levels of miR-144 in GC tissue may be associated with poor patient prognosis and it was demonstrated that the reintroduction of miR-144 increased the susceptibility of cancer cells to chemotherapy $(17,18)$. It has been reported that GATA4, a key regulator of cardiogenesis via cell autonomous or non-autonomous mechanisms, may be involved in the activation of miR-144 (19,20). Furthermore, the ability of miR-144 to inhibit the proliferation and metastasis of tumor cells is dependent on the target genes involved (21). Thus, the present study aimed to determine the role of miR-144 in $\mathrm{GC}$ tissues.

COX-2 is a rate-limiting enzyme, which converts arachidonic acid into prostaglandin. It has been demonstrated in vivo and in vitro that the expression of COX-2 is associated with the proliferation and metastasis of tumor cells (22). In addition, COX-2 has been proposed as a potential pharmacologic target to decrease the growth of certain human tumors (23). Previous studies identified that the expression of COX-2 was upregulated in GC and may be targeted by miR-143 $(24,25)$, which suggests that targeted COX-2 by miRNA may serve as a novel approach for the treatment of GC.

The present study demonstrated that the expression of miR-144 and GATA4 were downregulated in GC tissues and cell lines. Treatment with 5-aza-2'-dexoxycytidine (5-aza) upregulated the expression of miR-144 and GATA4 in MGC-803 cells. Overexpression of miR-144 and GATA4 was able to increase MGC-803 cell viability and colony formation, and miR-144 directly targeted and suppressed 
cyclooxygenase-2 (COX-2) expression. These results may improve understanding of the molecular mechanism of anti-oncogenic miRNAs in the initiation and progression of GC and provide evidence supporting the use of miRNAs to treat patients with GC.

\section{Materials and methods}

Cell lines and human cancer tissue samples. The human GC cell lines AGS, BGC-823, SGC-7901, MGC-803 and the normal gastric epithelium cell line GES-1 were obtained from the Institute of Biochemistry and Cell Biology at the Chinese Academy of Sciences (Shanghai, China). Cells were maintained in RPMI 1640 medium (Gibco; Thermo Fisher Scientific, Inc., Waltham, MA, USA) supplemented with $10 \%$ fetal bovine serum (Hyclone; GE Healthcare Life Sciences, Logan, UT, USA) and $1 \%$ penicillin/streptomycin, and cultured at $37^{\circ} \mathrm{C}$ in a humidified chamber supplemented with $5 \% \mathrm{CO}_{2}$. A total of 21 human GC tissue samples were obtained from patients undergoing surgical resection at Harbin Medical University Cancer Hospital (Harbin, China) between January 2014 and July 2015. There were 11 males and 10 females and the mean age of the patients was 46. Matched normal tissue samples were obtained from a segment of resected specimens that were $>5 \mathrm{~cm}$ from the edge of the tumor. Informed consents were obtained from all patients and the Research Ethics Committee of the Affiliated Tumor Hospital of Harbin Medical University approved the study.

Vector construction and transfection. A wild-type 3'-untranslated region (UTR) fragment of human COX-2 mRNA was amplified and cloned downstream of the pMIR-REPORT vector (Ambion; Thermo Fisher Scientific, Inc.) using HindIII and SpeI restriction sites to generate the pMIR-COX-2-3'-UTR luciferase vector (COX-2 3'-UTR). The mutation of the 3'-UTR fragment of human COX-2 mRNA was performed using the QuikChange mutagenesis kit (Stratagene; Agilent Technologies, Inc., Santa Clara, CA, USA) and cloned into the pMIR-REPORT vector to form the pMIR-COX-2-3'UTR-mut (COX-2-3'UTR-mut). To construct pcDNA3-GATA4, the fragment of GATA4 mRNA was amplified and cloned downstream of the pcDNA3.1 (Invitrogen; Thermo Fisher Scientific, Inc.). The miR-144 mimics (5'-UAC AGUAUAGAUGAUGUACU-3'), miR-144 antisense oligonucleotides (ASO; 5'-AGUACAUCAUCUAUACUGUA-3'), COX-2 small interfering (si)RNA and their corresponding controls [miRNA-negative control (NC), ASO NC and siRNA NC] were synthesized by Shanghai GenePharma Co., Ltd. (Shanghai, China). Transfections of miRNA mimics, ASO, siRNA and corresponding controls into MGC-803 cells were conducted using Lipofectamine ${ }^{\circledR} 2000$ (Invitrogen; Thermo Fisher Scientific, Inc.) according to the manufacturer's instructions using a final concentration of $50 \mathrm{nM}$ for RNA mimics, $100 \mathrm{nM}$ for ASO, $100 \mathrm{nM}$ for siRNA and their respective NCs. The COX-2 siRNA target sequence was 5'-GCTGGGAAG CCTTCTCTAA-3'; the siRNA sense strand was 5'-GCUGGG AAGCCUUCUCUAAdTdT-3' and the antisense strand was 5'-dTdTCGACCCUUCGGAAGAGAUU-3'.

Luciferase reporter assay. For the luciferase assay, MGC-803 cells $\left(1 \times 10^{5}\right.$ cells/well $)$ werecultured in triplicate in 48 -wellplates and then co-transfected with COX-23'UTR/COX-2-3'UTR-mut and miR-144 mimics/miRNA NC at the indicated concentrations using Lipofectamine 2000. After 48 h, a dual-luciferase reporter assay kit (Promega Corporation, Madison, WI, USA) was used to determine luciferase intensity. The pRL-TK and pMIR-vectors were used as internal controls and the results were expressed as the relative luciferase activity normalized to the Renilla luciferase acitivity.

5-Aza-CdR (5-Aza) treatment of GC cell line MGC-803. MGC-803 cells were treated with $3 \mu \mathrm{M}$ 5-Aza (cat. no. A3656; Sigma-Aldrich, Merck KGaA, Darmstadt, Germany) in RPMI 1640 medium (Gibco; Thermo Fisher Scientific, Inc.) supplemented with $10 \%$ fetal bovine serum (Hyclone; GE Healthcare Life Sciences) and 1\% penicillin/streptomycin for $48 \mathrm{~h}$ at $37^{\circ} \mathrm{C}$ in a humidified chamber supplemented with $5 \% \mathrm{CO}_{2}$. And the RT-qPCR was performed as follows.

$R N A$ preparation and reverse transcription-quantitative polymerase chain reaction $(R T-q P C R)$. RNA was extracted from tissues and cells using the mirVana miRNA isolation kit (Ambion; Thermo Fisher Scientific, Inc.) following the manufacturer's protocol. The separated and purified RNAs were detected by $1.5 \%$ agarose gel electrophoresis. Subsequently, $5 \mu \mathrm{g}$ RNA was reversely transcribed into cDNA using Moloney Murine Leukemia Virus Reverse Transcriptase (Invitrogen; Thermo Fisher Scientific, Inc.). 5x First-Strand Buffer and dNTPs were purchased from Invitrogen (Thermo Fisher Scientific, Inc.). The reverse transcription temperature protocol was $16^{\circ} \mathrm{C}$ for $30 \mathrm{~min} ; 42^{\circ} \mathrm{C}$ for $30 \mathrm{~min} ; 85^{\circ} \mathrm{C}$ for $5 \mathrm{~min}$, then hold at $4^{\circ} \mathrm{C}$. qPCR analysis was performed using the SYBR Premix Ex Taq ${ }^{\mathrm{TM}}$ kit (Takara Biotechnology Co., Ltd., Dalian, China) following the manufacturer's protocol. The PCR thermocycling conditions were as follows: 30 cycles of denaturation at $94^{\circ} \mathrm{C}$ for $30 \mathrm{sec}$, annealing at $58^{\circ} \mathrm{C}$ for $30 \mathrm{sec}$ and extension at $72^{\circ} \mathrm{C}$ for $30 \mathrm{sec}$. U6 (for miR-144) and GAPDH (for GATA4 and COX-2) were used an as internal control and the $2^{-\Delta \Delta \mathrm{Cq}}$ method (26) was used for quantification. The experiments were performed three times. The primers used are as follows: miR-144 forward, 5'-ATC CAGTGCGTGTCGTG-3' and reverse, 5'-TGCTTATACAGT ATAGATG-3'. U6 forward, 5'-CTCGCTTCGGCAGCACA-3' and reverse 5'-AACGCTTCACGAATTTGCGT-3'. GATA4 forward, 5'-CTGGCCTGTCATCTCACTACG-3' and reverse 5'-GGTCCGTGCAGGAATTTGAGG-3'. COX-2 forward, 5'-CCTGTGCCTGATGATTGC-3' and reverse 5'-CTGATG CGTGAAGTGCTG-3'. GAPDH forward, 5'-AATCCCATC ACCATCTTCCAGG-3' and reverse 5'-GAGTGGGTGTCG CTGTTGAAGT-3'.

Cell viability assay. An MTT assay was used to investigate the effect of GATA4 and miR-144 overexpression on cell viability. Briefly, cells were seeded at 1,000 cells/well and incubated at $37^{\circ} \mathrm{C}$ for $24 \mathrm{~h}$. Cells were then co-transfected with miR-144 mimics or pcDNA3-GATA4. Cells were harvested at following $2 \mathrm{~h}$ incubation with MTT $(0.5 \mathrm{mg} / \mathrm{ml})$ and dimethyl sulfoxide. The optical density was determined at $570 \mathrm{~nm}$ on a Tecan SpectraFluor microplate reader (Tecan Group Ltd., Männedorf, Switzerland). 
Colony formation assay. For the colony formation assay, cells transfected with miR-144 mimics or pcDNA3-GATA4 were seeded at 1,000 cells/well in a 6-well plate and incubated for 2 weeks at $37^{\circ} \mathrm{C}$ in a humidified chamber supplemented with $5 \% \mathrm{CO}_{2}$. The culture medium was replenished every 3 days and experiments were performed three times. The media was subsequently removed and fixed with $75 \%$ ethanol for $30 \mathrm{~min}$ at room temperature, $0.2 \%$ crystal violet stain (Beyotime Institute of Biotechnology, Haimen, China) was added to the wells for $20 \mathrm{~min}$ at room temperature, the plate was washed with running water and air dried. Then the colonies in each well were counted under a light microscope (magnification, x400; Olympus Corporation, Tokyo, Japan) following.

Cell cycle analysis. A total of $48 \mathrm{~h}$ after transfection, cells transfected with miR-144 mimics or pcDNA3-GATA4 were subjected to cell cycle analysis. Cells were obtained by trypsinization followed by centrifugation at $300 \mathrm{x}$ g for $5 \mathrm{~min}$. The cells were fixed with $70 \%$ ethanol on ice for $2 \mathrm{~h}$ followed by centrifuging for $5 \mathrm{~min}$ at $300 \mathrm{xg}$ at $4^{\circ} \mathrm{C}$. Subsequently, $0.05 \mathrm{mg} / \mathrm{ml}$ propidium iodide (PI; Sigma-Aldrich; Merck $\mathrm{KGaA}$ ) and $0.1 \mathrm{mg} / \mathrm{ml}$ RNAse A (Sigma-Aldrich; Merck $\mathrm{KGaA}$ ) were added to the samples for $30 \mathrm{~min}$. Cells were examined using a BD FACSCalibur flow cytometer (BD Biosciences, San Jose, CA, USA) and CellQuest software version 3.3 (BD Biosciences). The experiments were repeated three times.

Apoptosis assay. Annexin V-fluorescein isothiocyanate and PI staining were used to determine the effect of miR-144 mimics or pcDNA3-GATA4 on cell apoptosis. The adherent cells were harvested $48 \mathrm{~h}$ after transfection. Subsequently, cells were washed with PBS and stained with Annexin-V in combination with PI at room temperature in the dark for 15 min (Annexin V-PI kit; Nanjing KeyGen Biotech Co., Ltd., Nanjing, China). Cells were subsequently analyzed using a BD FACSCalibur flow cytometer (BD Biosciences) and CellQuest software version 3.3. A total of 10,000 events were counted for each sample.

Western blot analysis. Proteins from transfected cells were extracted using radioimmunoprecipitation assay lysis buffer (cat. no. R0278; 1:1,000; Sigma-Aldrich; Merck KGaA) and the protein concentration was determined using BCA assay kit (Thermo Fisher Scientific, Inc.). Equal amounts of protein (30 $\mu \mathrm{g} /$ lane) were separated by $12 \%$ SDS-PAGE followed by a transfer onto a nitrocellulose membrane. Then membranes were blocked in 5\% non-fat milk in TBS with $0.05 \%$ Tween-20 (TBST) at room temperature for $1 \mathrm{~h}$ and incubated with diluted primary antibodies [anti-COX-2 (cat. no. 4842; 1:1,000; Cell Signaling Technology, Inc., Danvers, MA, USA) and anti-GAPDH (cat. no. G9545; 1:1,000; Sigma-Aldrich; Merck KGaA)] overnight at $4^{\circ} \mathrm{C}$ and were subsequently washed three times in TBST, followed by incubation with horseradish peroxidase conjugated secondary antibodies (cat. no. AC111P; 1:1,000; Sigma-Aldrich; Merck KGaA) for $1 \mathrm{~h}$ at room temperature. The protein bands were detected with the Western Blotting Luminol reagent (cat. no. sc-2048; Santa Cruz Biotechnology, Inc., Dallas, TX, USA) and quantified by densitometric analysis of digitized autoradiograms using Quantity One version 4.6.2 software (Bio-Rad Laboratories, Inc., Hercules, CA, USA).

Bioinformatics method, 3'-UTR datasets and microRNA datasets. Mir-144 target sites were predicted by computer-aided algorithms obtained from Targetscan Release 7.1 (targetscan.org/). The 3'-UTR sequences of COX-2 were retrieved using the Ensembl database (ensembl.org). Human miRNA sequences of the miR-144 registry were downloaded from the miRBase website (mirbase.org).

Statistical analysis. Statistical analysis was performed using a two-tailed Student's t-test on SPSS 17.0 software (SPSS Inc., Chicago, IL, USA). For comparison of multiple groups, one-way analysis of variance followed by a Tukey's post hoc multiple comparisons test was performed. All results are expressed as the mean \pm standard error of the mean. $\mathrm{P}<0.05$ was considered to indicate a statistically significant difference.

\section{Results}

miR-144/GATA4 is downregulated in human GC tissues and cell lines. RT-qPCR was performed to measure the expression of miR-144 and GATA4 in 21 pairs of GC and adjacent normal tissues taken from patients with GC. The results revealed that the expression of miR-144 and GATA4 were significantly decreased in GC tissues compared with the adjacent normal tissues (Fig. 1A). Subsequently, the expression of miR-144 and GATA4 in the GC cell lines was determined. The results from RT-qPCR indicated that the expression of miR-144 and GATA4 were significantly downregulated in the GC cell lines (MGC-803, SGC-7901, BGC-823 and AGS) relative to the normal cell line, GES-1 (Fig. 1B). As the expression of miR-144 was expressed at lowest level in the MGC-803 cell line, it was selected for miR-144 overexpression and associated assays.

Synergistic effects of GATA4 and miR-144 on cell viability and growth. Studies have indicated that GATA4 is able to transcriptionally activate miR-144; additionally GATA4 may potentially be involved in promoting the hypermethylation of tumor suppressor genes underlying tumorigenesis $(19,27,28)$. The results of the present study implied that DNA demethylation following treatment with 5-aza induced the upregulation of GATA4 and miR-144 expression compared with the cells without 5-aza treatment (Fig. 2A). Subsequently, miR-144 mimics/miRNA NC were transfected into cells and the expression of miR-144 was determined by RT-qPCR. The results confirmed that the overexpression of GATA4 upregulated miR-144 expression (Fig. 2B). In order to test the effects of GATA4 and miR-144 on cell viability, pcDNA3-GATA4 or miR-144 mimics were transfected into MGC-803 cells. It was revealed that overexpression of GATA4 and miR-144 induced time-dependent inhibition of cell viability (Fig. 2C). Furthermore, the results of the cell colony assay exhibited a similar inhibition on cell proliferation (Fig. 2D and E). This evidence indicates that GATA4 and miR-144 are potentially hypermethylated in GC, and that GATA4 and miR-144 inhibit the viability of GC cells. 
A

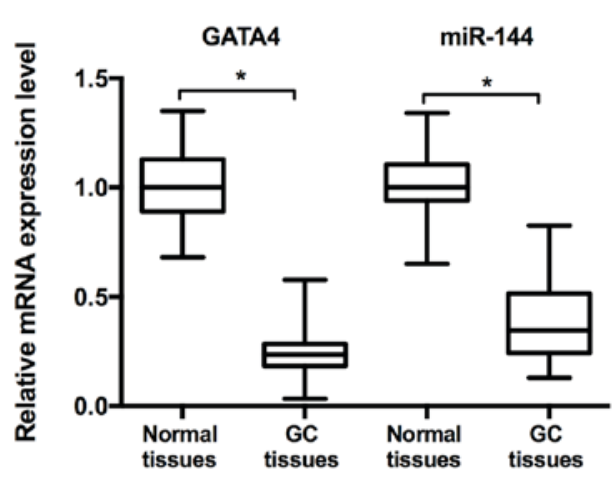

B

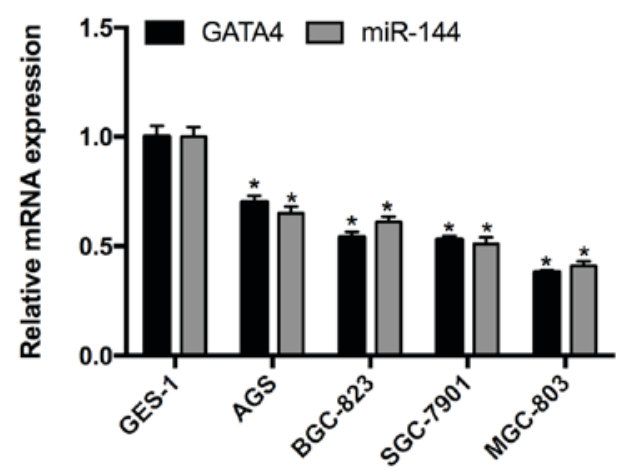

Figure 1. Expression of miR-144 and GATA4 in tissues and cell lines. (A) Relative levels of miR-144 and GATA4 in 21 patients with GC. * P<0.05 (B) Relative levels of miR-144 and GATA4 in the GC cell lines MGC-803, SGC-7901, BGC-823 and AGS and the normal cell line GES-1; ${ }^{*}<0.05$ vs. GES-1 cell line. miR-144, microRNA-144; GC, gastric cancer.

GATA4 and miR-144 induce GC cell cycle arrest and apoptosis. To investigate whether GATA4 and miR-144 affect the cell cycle and apoptosis of GC cells, fluorescence-activated cell sorting and a cell apoptosis assay were performed. The results revealed that overexpression of GATA4 increased the proportion of cells in the G1 phase and concurrently decreased the proportion of cells in the S-phase compared with the control (Fig. 3A). Transfection with miR-144 mimic had a similar effect on the cell cycle. In accordance with this, elevated expression of GATA4/miR-144 mimics significantly promoted apoptosis (Fig. 3B). Altogether, the results indicated that the elevated expression of GATA4/miR-144 may induce $\mathrm{GC}$ cell cycle arrest and apoptosis.

miR-144 negatively regulates $C O X-2$, which inhibits $G C$ cell viability and growth. Although it has been demonstrated that miR-144 targets COX-2 in esophageal squamous cell cancer (29), there have been few studies investigating whether miR-144 regulates COX-2 in GC. Bioinformatics prediction using Targetscan Release 7.1 (targetscan.org/) was performed and COX was confirmed to be a direct target of miR-144 (Fig. 4A). The luciferase intensity of MGC-803 cells transfected with miR-144 mimics+COX-2-3'-UTR was significantly lower than the other three groups (miRNA NC+COX-2-3'-UTR, miRNA NC+COX-2-3'-UTR-mut and miR-144+COX-2-3'-UTR-mut), indicating that miR-144 may directly bind to COX-2 3'-UTR (Fig. 4B). The possibility that miR-144 serves a functional role in the regulation of endogenous COX-2 expression in GC cells was also assessed. The results of RT-qPCR revealed that cells transfected with miR-144 mimic exhibited a significantly decreased expression of endogenous COX-2 compared with the miRNA NC group (Fig. 4C). The results of the western blot analysis also exhibited a similar inhibitory effect (Fig. 4D). To further explore the role of COX-2 in the proliferation of $\mathrm{GC}$ cells, the expression of COX-2 was inhibited following transfection of COX-2 siRNA or siRNA NC. The results obtained from the MTT assay revealed that the viability of MGC-803 cells decreased in a time-dependent manner (Fig. 4E). The results of the colony formation assay further determined that inhibition of COX-2 reduced cell viability (Fig. 4F). These results indicate that miR-144 directly targets COX-2 3'UTR to suppress its expression and inhibit GC cell viability and growth.

Synergistic effects of GATA4 and miR-144 on COX-2 expression in $G C$ cells. RT-qPCR and western blotting were performed to further investigate whether GATA4 and miR-144 are able to regulate COX-2 expression. The results revealed that the overexpression of GATA4 (pcDNA3-GATA4+ASO $\mathrm{NC}$ ) significantly reduced the expression of COX-2 mRNA and protein, whereas miR-144 ASO (pcDNA3+miR-144 ASO) promoted the expression of COX-2 mRNA and protein (Fig. 5). Additionally, the reduction induced by pcDNA3-GATA4 can be partially rescued by miR-144 ASO (pcDNA3-GATA4+miR-144 ASO) compared with the pcDNA3+ASO NC group (Fig. 5). These results indicate that GATA4 and miR-144 exhibit a synergistic effect on the inhibition of COX-2 expression in GC cells.

\section{Discussion}

The mortality rate of patients with GC is increasing in developing countries, particularly in East Asian countries (30). The overall 5-year survival rate remains low; ranging between $20-30 \%$ for patients past the early stage of the disease at the time of their initial diagnosis (31). Furthermore, the dysregulation of miRNAs is reported to be involved in the pathogenic processes of GC tumorigenesis by regulating the expression of the miRNA's target genes. It has been revealed that the regulation of miRNAs participating in GC tumorigenesis is associated with cell proliferation and apoptosis-related genes (32). Therefore, numerous studies have suggested that miRNAs, including miR-10b, miR-106a and miR-107, may be used as biomarkers in GC $(14,33,34)$. In addition, it has been demonstrated that gene silencing is tightly associated with epigenetic mechanisms, particularly promoter hypermethylation of tumor suppressor genes, which may serve a critical role in the mechanisms underlying tumorigenesis $(27,28)$.

In the present study, it was revealed that the expression of GATA4 and miR-144 was repressed in GC tissues and cell lines compared with relative controls. It was hypothesized that the downregulation of GATA4 and miR-144 that occurs in $\mathrm{GC}$ is induced by hypermethylation. Consistent with this, 


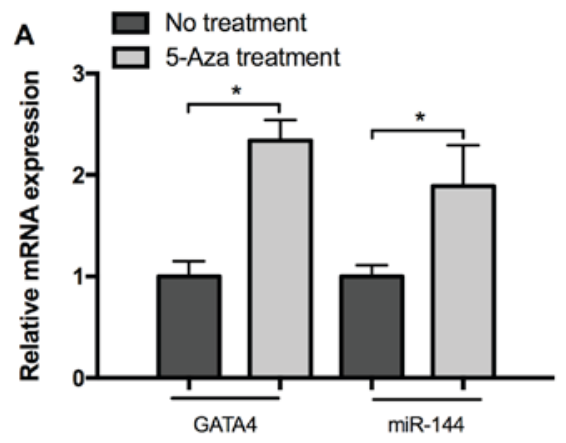

B
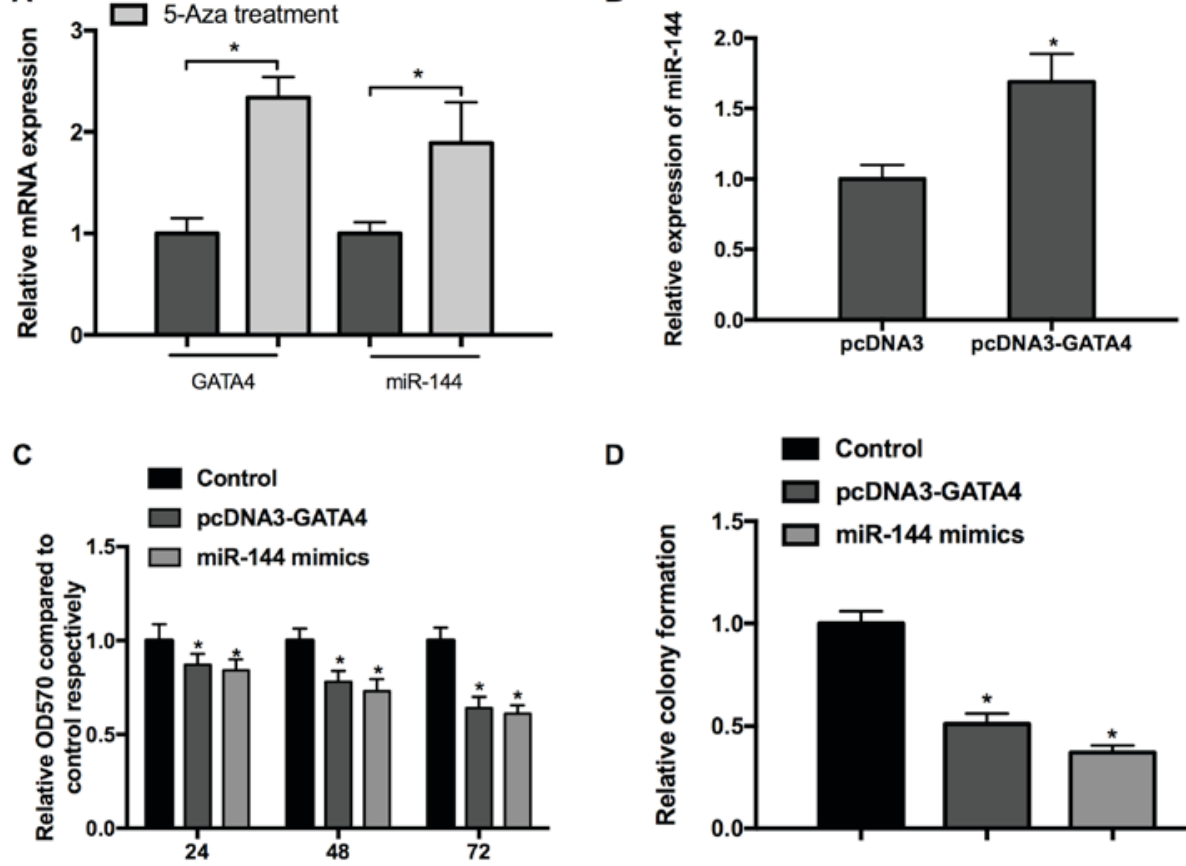

D

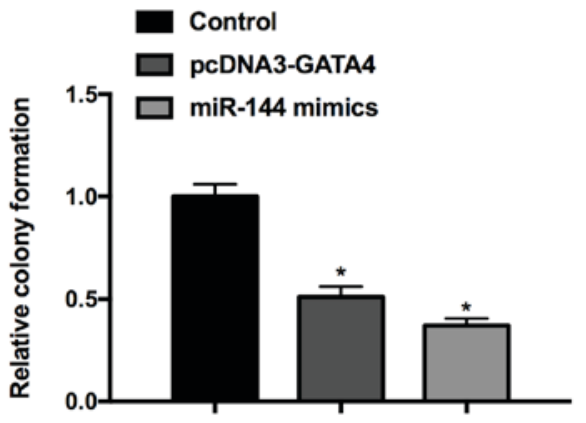

E

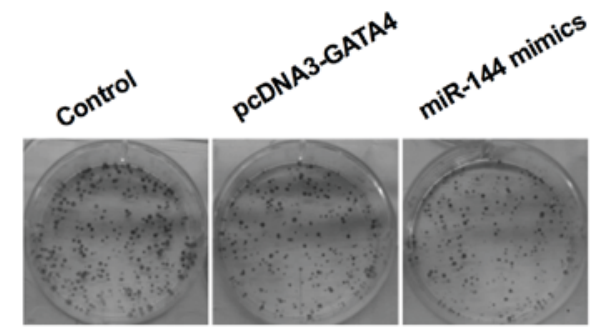

Figure 2. Synergistic effects of GATA4 and miR-144 on cell viability and growth (A) Treatment with 5-aza increased the expression of GATA4 and miR-144 (B) The expression of miR-144 was promoted in cells transfected with pcDNA3-GATA4. (C) GATA4 and miR-144 inhibited cell viability in a time-dependent manner. (D and E) GATA4 and miR-144 inhibited cell growth, as demonstrated by the results of a cell colony assay. "P<0.05 vs. corresponding control. 5-aza, 5-aza-2'-dexoxycytidine; miR-144, microRNA-144.
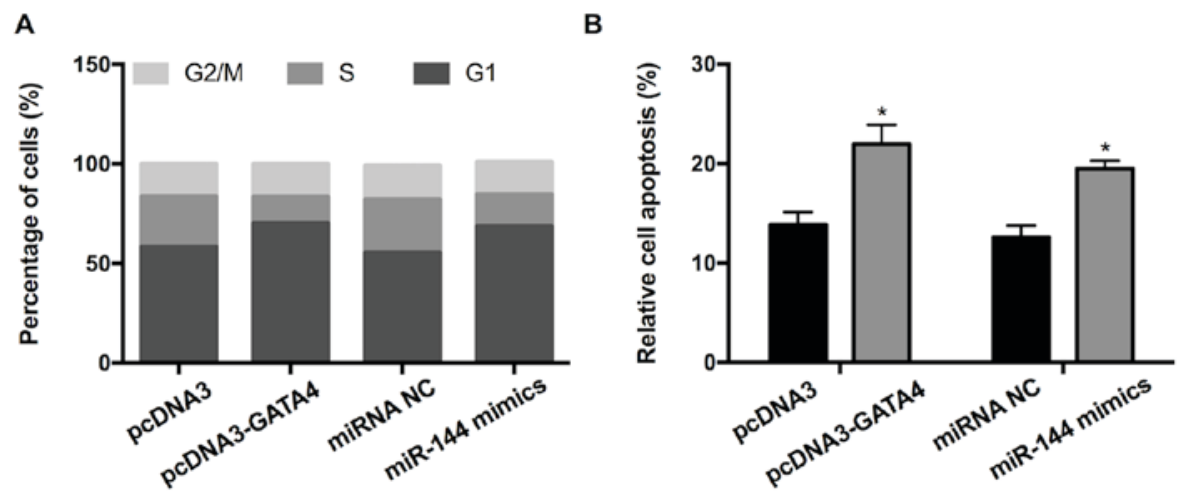

Figure 3. Effect of GATA4 and miR-144 on the cell cycle and apoptosis. (A) Fluorescence-activated cell sorting analysis of cells transfected with pcDNA-GATA4, miR-144 mimics and corresponding controls. (B) Analysis of apoptosis in cells transfected with pcDNA-GATA4, miR-144 mimics and corresponding controls; ${ }^{*} \mathrm{P}<0.05$ vs. corresponding control. miR-144, microRNA-144.

the results of the present study indicated that cells treated with 5-aza exhibited increased expression of GATA4 and miR-144. It has been demonstrated that dysregulated cell proliferation and the induction of cell cycle arrest are key mechanisms involved in tumor progression (35). Furthermore, miR-144 and GATA4 may function by modulating the expression of genes that suppress tumor growth (36-38). The results of the MTT and colony formation assays validated this and revealed that 
A

\begin{tabular}{|c|c|}
\hline COX-2 3' UTR & $5^{\prime}$...AAAAACAAAACCAAAUGAUAUCU... \\
\hline hsa-miR-144 & 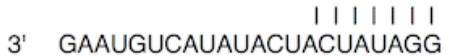 \\
\hline & $\begin{array}{c}11111 \\
51 . \\
\text { 5AAAACAAAACCAAAUCAUAUG }\end{array}$ \\
\hline
\end{tabular}

B
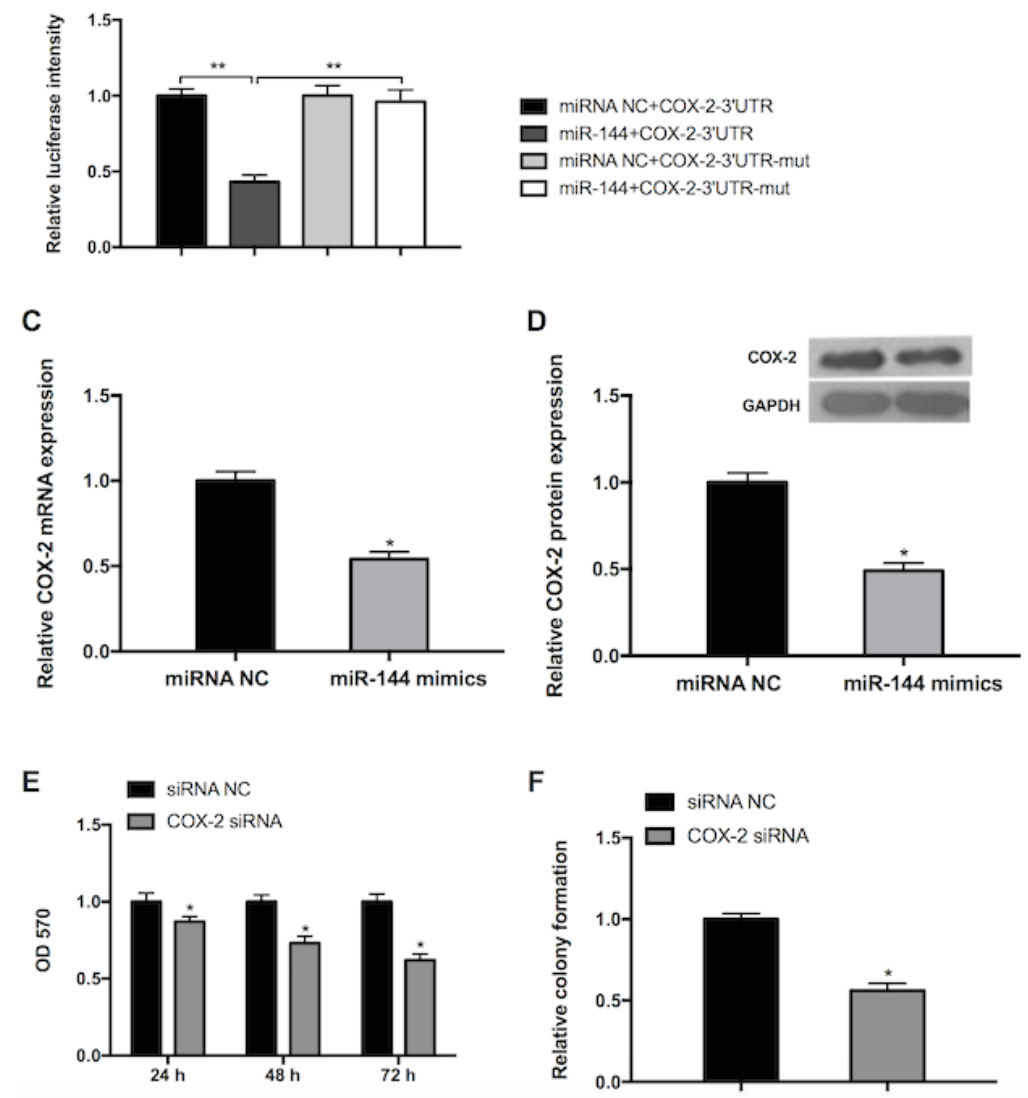

Figure 4. miR-144 targets COX-2, which inhibits the viability and growth of GC cells. (A) A schematic of the bioinformatics prediction in the 3'-UTR of COX-2 as well as mutant 3'-UTR used in the present study. (B) The results of the luciferase reporter assay demonstrated that COX-2 is a direct target of miR-144. ${ }^{* *} \mathrm{P}<0.01$. The expression of COX-2 was reduced in cells transfected with miR-144 mimics, as determined by (C) reverse transcription-quantitative polymerase chain reaction and (D) western blot analysis. (E) Knockdown of COX-2 reduced cell viability in a time-dependent manner, as determined by the results of an MTT assay. (F) Knockdown of COX-2 inhibited cell growth, as determined by the results of a cell colony formation assay. Differences were assessed by one-way analysis of variance followed by Tukey's multiple comparison test. "P $<0.05$ vs. corresponding control. miR-144, microRNA-144; COX-2, cyclooxygenase-2; UTR, untranslated region; mut, mutant; NC, negative control; siRNA, small interfering RNA; OD, optical density.

A

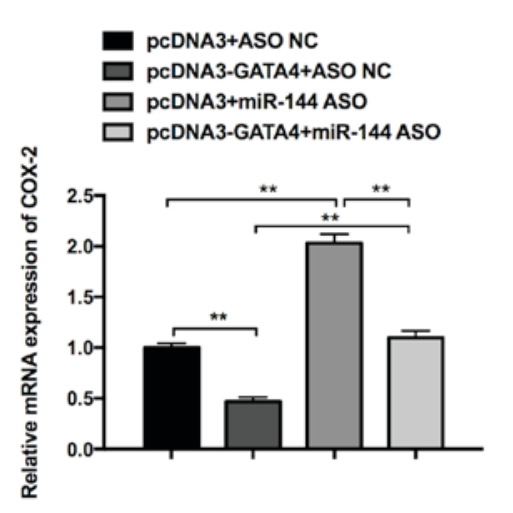

B

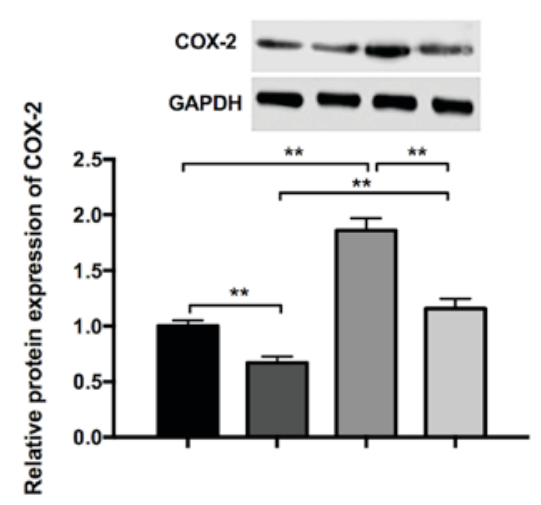

Figure 5. Synergistic effects of GATA4 and miR-144 on COX-2 expression in gastric cancer cells. (A) Expression of COX-2 in cells transfected with pcDNA3+ASO NC, pcDNA3-GATA4+ASO NC, pcDNA3+miR-144 ASO, pcDNA3-GATA4+miR-144 ASO, as determined by reverse transcription-quantitative polymerase chain reaction. (B) Expression of COX-2 in cells transfected with pcDNA3+ASO NC, pcDNA3-GATA4+ASO NC, pcDNA3+miR-144 ASO, pcDNA3-GATA4+miR-144 ASO, as determined by western blot analysis. Differences were assessed using one-way analysis of variance followed by Tukey's multiple comparison test. ${ }^{* *} \mathrm{P}<0.01$. miR-144, microRNA-144; COX-2, cyclooxygenase-2; ASO, antisense oligonucleotides; NC, negative control. 
miR-144 and GATA4 have a synergistic effect on cell proliferation, apoptosis and the cell cycle.

To further identify the molecular mechanism of miR-144 in the regulation of cancer progression, a bioinformatics analysis was performed, which indicated that COX-2 is a potential target of miR-144. COX-2 is the rate-limiting enzyme involved in the biosynthesis of prostaglandins and it has been suggested that COX-2 is involved in the development and progression of tumors $(39,40)$. In the present study, knockdown of COX-2 expression by siRNA in MGC-803 cells decreased cell viability and colony formation. Furthermore, the results of the luciferase reporter assay validated that miR-144 targets the 3'UTR of COX-2 and GATA4 and that miR-144 has a synergistic effect on the regulation of COX-2 in GC cells.

In conclusion, the results of the present study revealed that GATA4 and miR-144 are downregulated in GC and that this may be attributed to hypermethylation. Furthermore, GATA4 and miR-144 had a synergistic effect on cell proliferation and apoptosis, potentially by targeting COX-2. These results indicate that GATA4 and miR-144 inhibit the proliferation of GC cells and that they may be used as a potential therapeutic target in the treatment of GC.

\section{Acknowledgements}

The present study was supported by the Health and Life Committee Fund of Heilongjiang Province (grant no. 2016-111) and the Applied Technology Research and Development Project of Harbin, Heilongjiang Province (grant no. 2016RAQXJ152).

\section{References}

1. Heise K, Bertran E, Andia ME and Ferreccio C: Incidence and survival of stomach cancer in a high-risk population of Chile. World J Gastroenterol 15: 1854-1862, 2009.

2. Yang L: Incidence and mortality of gastric cancer in China. World J Gastroenterol 12: 17-20, 2006.

3. Shi C, Liu B, Yan J, Liu H, Pan Z, Yao W, Yan F and Zhang H: Gastric cancer: Preoperative TNM staging with individually adjusted computed tomography scanning phase. J Comput Assist Tomogr 40: 160-166, 2016

4. Hu B, El Hajj N, Sittler S, Lammert N, Barnes R and Meloni-Ehrig A: Gastric cancer: Classification, histology and application of molecular pathology. J Gastrointest Oncol 3: 251-261, 2012

5. Smyth EC and Cunningham D: Gastric cancer in 2012: Defining treatment standards and novel insights into disease biology. Nat Rev Clin Oncol 10: 73-74, 2013.

6. Dassen AE, Lemmens VE, van de Poll-Franse LV, Creemers GJ, Brenninkmeijer SJ, Lips DJ, Vd Wurff AA, Bosscha K and Coebergh JW: Trends in incidence, treatment and survival of gastric adenocarcinoma between 1990 and 2007: A population-based study in the Netherlands. Eur J Cancer 46: 1101-1110, 2010.

7. Wu CW, Lo SS, Shen KH, Hsieh MC, Lui WY and P'eng FK: Surgical mortality, survival and quality of life after resection for gastric cancer in the elderly. World J Surg 24: 465-472, 2000.

8. Yalçin S: The increasing role of pharmacogenetics in the treatment of gastrointestinal cancers. Gastrointest Cancer Res 3: 197-203, 2009

9. Melo SA and Esteller M: Dysregulation of microRNAs in cancer: Playing with fire. FEBS Lett 585: 2087-2099, 2011.

10. Wang YY, Li L, Ye ZY, Zhao ZS and Yan ZL: MicroRNA-10b promotes migration and invasion through Hoxd10 in human gastric cancer. World J Surg Oncol 13: 259, 2015.

11. Katada T, Ishiguro H, Kuwabara Y, Kimura M, Mitui A, Mori Y, Ogawa R, Harata K and Fujii Y: microRNA expression profile in undifferentiated gastric cancer. Int J Oncol 34: 537-542, 2009.
12. Ueda T, Volinia S, Okumura H, Shimizu M, Taccioli C, Rossi S, Alder H, Liu CG, Oue N, Yasui W, et al: Relation between microRNA expression and progression and prognosis of gastric cancer: A microRNA expression analysis. Lancet Oncol 11: 136-146, 2010.

13. Kim YK, Yu J, Han TS, Park SY, Namkoong B, Kim DH, Hur K, Yoo MW, Lee HJ, Yang HK and Kim VN: Functional links between clustered microRNAs: Suppression of cell-cycle inhibitors by microRNA clusters in gastric cancer. Nucleic Acids Res 37: 1672-1681, 2009.

14. Li X, Zhang Y, Shi Y, Dong G, Liang J, Han Y, Wang X, Zhao Q, Ding J, Wu K and Fan D: MicroRNA-107, an oncogene microRNA that regulates tumour invasion and metastasis by targeting DICER1 in gastric cancer. J Cell Mol Med 15: 1887-1895, 2011.

15. Jiang H, Yu WW, Wang LL and Peng Y: miR-130a acts as a potential diagnostic biomarker and promotes gastric cancer migration, invasion and proliferation by targeting RUNX3. Oncol Rep 34: $1153-1161,2015$

16. Chen Q, Ge X, Zhang Y, Xia H, Yuan D, Tang Q, Chen L, Pang X, Leng W and Bi F: Plasma miR-122 and miR-192 as potential novel biomarkers for the early detection of distant metastasis of gastric cancer. Oncol Rep 31: 1863-1870, 2014.

17. Meder B, Backes C, Haas J, Leidinger P, Stähler C, Großmann T, Vogel B, Frese K, Giannitsis E, Katus HA, et al: Influence of the confounding factors age and sex on microRNA profiles from peripheral blood. Clin Chem 60: 1200-1208, 2014.

18. Imaoka H, Toiyama Y, Okigami M, Yasuda H, Saigusa S, Ohi M, Tanaka K, Inoue Y, Mohri Y and Kusunoki M: Circulating microRNA-203 predicts metastases, early recurrence and poor prognosis in human gastric cancer. Gastric Cancer 19: 744-753, 2016.

19. Akiyoshi S, Fukagawa T, Ueo H, Ishibashi M, Takahashi Y, Fabbri M, Sasako M, Maehara Y, Mimori K and Mori M: Clinical significance of miR-144-ZFX axis in disseminated tumour cells in bone marrow in gastric cancer cases. Br J Cancer 107: 1345-1353, 2012.

20. Liu J, Xue H, Zhang J, Suo T, Xiang Y, Zhang W, Ma J, Cai D and Gu X: MicroRNA-144 inhibits the metastasis of gastric cancer by targeting MET expression. J Exp Clin Cancer Res 34: $35,2015$.

21. Grépin C, Nemer G and Nemer M: Enhanced cardiogenesis in embryonic stem cells overexpressing the GATA-4 transcription factor. Development 124: 2387-2395, 1997.

22. Zhang X, Wang X, Zhu H, Zhu C, Wang Y, Pu WT, Jegga AG and Fan GC: Synergistic effects of the GATA-4-mediated miR-144/451 cluster in protection against simulated ischemia/reperfusion-induced cardiomyocyte death. J Mol Cell Cardiol 49: 841-850, 2010.

23. Yin J, Liu B, Li B, Liu Z, Xie X, Lv Z, Gao S and Guang J: The cyclooxygenase-2 inhibitor celecoxib attenuates hepatocellular carcinoma growth and c-Met expression in an orthotopic mouse model. Oncol Res 19: 131-139, 2011.

24. Wu XL, Cheng B, Li PY, Huang HJ, Zhao Q, Dan ZL, Tian DA and Zhang P: MicroRNA143 suppresses gastric cancer cell growth and induces apoptosis by targeting COX-2. World $\mathrm{J}$ Gastroenterol 19: 7758-7765, 2013.

25. Wang Z, Chen JQ and Liu JL: COX-2 inhibitors and gastric cancer. Gastroenterol Res Pract 2014: 132320, 2014.

26. Livak KJ and Schmittgen TD: Analysis of relative gene expression data using real-time quantitative PCR and the 2(-Delta Delta C(T)) method. Methods 25: 402-408, 2001.

27. Lynch J, Meehan MH, Crean J, Copeland J, Stallings RL and Bray IM: Metastasis suppressor microRNA-335 targets the formin family of actin nucleators. PLoS One 8: e78428, 2013.

28. Ma J, Hong L, Chen Z, Nie Y and Fan D: Epigenetic regulation of microRNAs in gastric cancer. Dig Dis Sci 59: 716-723, 2014.

29. Shao Y, Li P, Zhu ST, Yue JP, Ji XJ, Ma D, Wang L, Wang YJ, Zong Y, Wu YD and Zhang ST: MiR-26a and miR-144 inhibit proliferation and metastasis of esophageal squamous cell cancer by inhibiting cyclooxygenase-2. Oncotarget 7: 15173-15186, 2016.

30. Hsu KW, Hsieh RH, Huang KH, Fen-Yau Li A, Chi CW, Wang TY, Tseng MJ, Wu KJ and Yeh TS: Activation of the Notch1/STAT3/Twist signaling axis promotes gastric cancer progression. Carcinogenesis 33: 1459-1467, 2012.

31. Yu JW, Wu JG, Zheng LH, Zhang B, Ni XC, Li XQ and Jiang BJ: Influencing factors and clinical significance of the metastatic lymph nodes ratio in gastric adenocarcinoma. J Exp Clin Cancer Res 28: 55, 2009. 
32. Tsai MM, Wang CS, Tsai CY, Huang HW, Chi HC, Lin YH, $\mathrm{Lu} \mathrm{PH}$ and Lin KH: Potential diagnostic, prognostic and therapeutic targets of MicroRNAs in human gastric cancer. Int J Mol Sci 17: E945, 2016.

33. Hersey P and Zhang XD: Treatment combinations targeting apoptosis to improve immunotherapy of melanoma. Cancer Immunol Immunother 58: 1749-1759, 2009.

34. Xiao B, Guo J, Miao Y, Jiang Z, Huan R, Zhang Y, Li D and Zhong J: Detection of miR-106a in gastric carcinoma and its clinical significance. Clin Chim Acta 400: 97-102, 2009.

35. Evan GI and Vousden KH: Proliferation, cell cycle and apoptosis in cancer. Nature 411: 342-348, 2001.

36. Zhang Z, Kong Y, Yang W, Ma F, Zhang Y, Ji S, Ma EM, Liu H, Chen Y and Hua Y: Upregulation of microRNA-34a enhances the DDP sensitivity of gastric cancer cells by modulating proliferation and apoptosis via targeting MET. Oncol Rep 36: 2361-2397, 2016.
37. Song B, Yan J, Liu C, Zhou H and Zheng Y: Tumor suppressor role of miR-363-3p in gastric cancer. Med Sci Monit 21: 4074-4080, 2015.

38. Li D, Li Z, Xiong J, Gong B, Zhang G, Cao C, Jie Z, Liu Y, Cao Y, Yan Y, et al: MicroRNA-212 functions as an epigenetic-silenced tumor suppressor involving in tumor metastasis and invasion of gastric cancer through down-regulating PXN expression. Am J Cancer Res 5: 2980-2997, 2015.

39. Adhim Z, Matsuoka T, Bito T, Shigemura K, Lee KM, Kawabata M, Fujisawa M, Nibu K and Shirakawa T: In vitro and in vivo inhibitory effect of three Cox-2 inhibitors and epithelial-to-mesenchymal transition in human bladder cancer cell lines. Br J Cancer 105: 393-402, 2011.

40. Elmets CA, Ledet JJ and Athar M: Cyclooxygenases: Mediators of UV-induced skin cancer and potential targets for prevention. J Invest Dermatol 134: 2497-2502, 2014. 